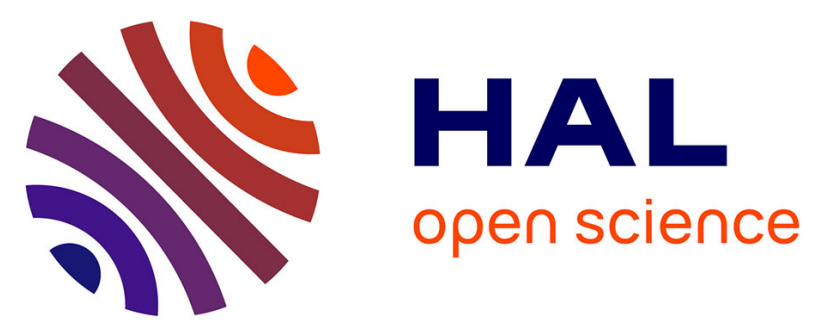

\title{
Randomized controlled trial of a 12-month computerized mindfulness-based intervention for obese patients with binge eating disorder: The MindOb study protocol
}

\author{
Alexis Ruffault, Claire Carette, Kàtia Lurbe I Puerto, Nicolas Juge, Alain
}

Beauchet, Jean-Jacques Benoliel, Jean-Marc Lacorte, Jean F. Fournier, Sébastien Czernichow, Cécile Flahault

\section{To cite this version:}

Alexis Ruffault, Claire Carette, Kàtia Lurbe I Puerto, Nicolas Juge, Alain Beauchet, et al.. Randomized controlled trial of a 12-month computerized mindfulness-based intervention for obese patients with binge eating disorder: The MindOb study protocol. Contemporary Clinical Trials, 2016, 49, pp.126-133. 10.1016/j.cct.2016.06.012 . hal-01343387

\section{HAL Id: hal-01343387 \\ https://hal.science/hal-01343387}

Submitted on 8 Jul 2016

HAL is a multi-disciplinary open access archive for the deposit and dissemination of scientific research documents, whether they are published or not. The documents may come from teaching and research institutions in France or abroad, or from public or private research centers.
L'archive ouverte pluridisciplinaire $\mathbf{H A L}$, est destinée au dépôt et à la diffusion de documents scientifiques de niveau recherche, publiés ou non, émanant des établissements d'enseignement et de recherche français ou étrangers, des laboratoires publics ou privés. 
Randomized controlled trial of a 12-month computerized mindfulness-based intervention for obese patients with binge-eating disorder: The MindOb study protocol

Alexis Ruffault ${ }^{1,2,3, *}$, Claire Carette ${ }^{2,3}$, Kàtia Lurbe i Puerto ${ }^{2,3}$, Nicolas Juge ${ }^{4}$, Alain Beauchet ${ }^{5}$, Jean-Jacques Benoliel ${ }^{6}$, Jean-Marc Lacorte ${ }^{6}$, Jean F. Fournier ${ }^{7}$, Sébastien Czernichow ${ }^{2,3,8,9}$, \& Cécile Flahault ${ }^{1}$

${ }^{1}$ Laboratoire de Psychopathologie et Processus de Santé (EA 4057), Université Paris Descartes, Sorbonne Paris Cité, France

${ }^{2}$ Unité de Nutrition, Hôpital Ambroise Paré, Assistance Publique-Hôpitaux de Paris, France

${ }^{3}$ Unité de Nutrition, Hôpital Européen Georges-Pompidou, Assistance Publique-Hôpitaux de Paris, France

${ }^{4}$ Mindeval, Canada

${ }^{5}$ Unité de Recherche Clinique, Hôpital Ambroise Paré, Assistance Publique-Hôpitaux de Paris, France

${ }^{6}$ Service de Biochimie Endocrinienne et Oncologique, HU Pitié-Salpêtrière-Charles Foix, Assistance Publique-Hôpitaux de Paris, France

${ }^{7}$ Université Paris Ouest Nanterre la Défense, France

${ }^{8}$ Université Versailles St Quentin en Yvelines, France

${ }^{9}$ INSERM UMS 011, Population-based cohorts, France

* Corresponding author:

Alexis Ruffault

Laboratoire de Psychopathologie et Processus de Santé (EA 4057)

Institut de Psychologie, Université Paris Descartes

71 avenue Edouard Vaillant

92100 Boulogne-Billancourt

France

alexis.ruffault@parisdescartes.fr

+33(0)176533107

Acknowledgements

Fondation Nestlé France for funding the research.

\section{Conflict of Interest Statement}

No conflict of interest is declared. 


\begin{abstract}
Background: Mindfulness-based interventions for healthy behaviors such as exercise and dietary modifications have aroused growing interest. This study aims to test the effectiveness of a mindfulness-based intervention for the reduction of impulsive eating and the improvement of motivation to exercise among obese individuals. Methods: One-hundredand-twenty obese outpatients, aged 18 to 65 years, diagnosed with a binge-eating disorder, will be randomly assigned to one of the three following groups: mindfulness practice, sham meditation or treatment as usual control. The tested intervention consists of a 1-year computerized mindfulness-based program. Mindfulness sessions are audio recordings that the patients are asked to listen to, 10 minutes every day. Self-reported questionnaires measuring impulsive eating, motivation to exercise, physical activity level, mood, and mindfulness skills are filled in at baseline, 1, 6 and 12 months. Physical activity, calories consumption and biomarkers are measured with more objective measurement tools at baseline, 6 months and 12 months. Conclusion: Mindfulness, as both a de-automation element and as a moderator of motivation to exercise, can lead to the reduction of impulsive eating, and also to an increase in levels of physical activity. These effects could cause weight loss in obese patients suffering from binge-eating disorder.
\end{abstract}

Trial registration: clinicaltrials.gov: NCT02571387.

Keywords: mindfulness, internet-based intervention, obesity, impulsive eating, physical activity 


\section{INTRODUCTION}

As many as $36.9 \%$ of men and $38.0 \%$ of women are overweight or obese in the world [1]. The World Health Organization (WHO) [2] estimates that obesity is the result of an inappropriate balance between energy intake and energy expenditure. Binge Eating Disorder (BED) is one of the leading causes of increased energy intake [2], and is characterized by recurrent and persistent episodes of impulsive (i.e., uncontrolled) eating sustained by distress without any compensatory behavior. Moreover, only one third of individuals with BED benefit from treatment [3]. While psychological or behavioral treatments aiming at reducing binge eating have little effect on weight loss and weight loss treatments are less effective in individuals with BED than those without BED [4], interventions with a broader range of applications could be of interest for effective long-term weight management. Additionally, while excess weight is associated with lower physical activity (PA) levels [2], prescribed exercise programs and information about PA need to be associated with psychological and motivational techniques to foster longer-term autonomous practice [5, 6]. Moreover, an increased PA level has been shown to temper brain responses to visual food cues, increasing capacity to regulate food intake [7].

Mindfulness is a non-judgmental and present-oriented awareness leading to acceptance of thoughts, emotions and sensations occurring in the present moment [8]. Over the last decade, research has shown a growing interest in mindfulness in the context of health behaviors [9]. Moreover, recent reviews and meta-analyses have investigated the effects of mindfulness-based interventions on weight loss and eating disorders in overweight and obese individuals [10-14]. While mindfulness appears to reduce eating disorders significantly (and more precisely, BED), its long-term efficacy on weight loss remains unknown. One explanation could be the duration of such interventions (mainly less than 3 months), which could be too short to integrate mindfulness into everyday life and observe a decrease in Body Mass Index (BMI). Another explanation could be the focus of interventions. In fact, interventions conducted with overweight and obese individuals mainly focus on eating behaviors, limiting the promotion of a "mindful lifestyle" [15] and thus the effects of mindfulness-based interventions on a multifactorial weight loss program. While it is known that obesity is caused by a wide range of factors [16] and associated with psychological comorbidities such as mood disorders, mindfulness training could be beneficial for obese individuals as a complementary and transdiagnostic approach [17].

Studies have shown that obese individuals tend to be more impulsive than normal weight individuals, suggesting that the tendency to over-eat without thinking is associated with impulsive personality [18-20]. Brief mindfulness training has shown an overall effect in reducing chocolate craving [21], as well as more self-controlled and risk-averse discounting patterns for food [22]. One explanation for the effectiveness of mindfulness training on impulsive eating could be that increased attention to present moment experience could change tendencies to be over-responsive to food cues, fostering healthier decision-making [23]. Ulmer and colleagues [24] and Butryn and colleagues [25] showed that higher mindfulness skills are linked to higher PA levels and maintenance, and that mindfulness training could increase PA levels, respectively. These links have been studied in cross-sectional studies aiming at understanding the mechanisms of change related to higher mindfulness skills, in the context of PA behavior [26-28]. These studies emphasized the moderating role of mindfulness skills in the behavior change process for increasing PA in healthy individuals.

The purpose of this study is to evaluate the effectiveness of a daily 12 -month computerized mindfulness-based intervention in obese patients diagnosed with BED. The intervention aims at increasing mindfulness skills in obese patients in order to help them manage their impulsive eating and motivation towards PA. The intervention is thought of as complementary to medical and paramedical care provided to obese patients. The self-help 
design has been chosen so that patients will not have to go to the hospital on a frequent basis, and to release staff (e.g., psychologists) from supplementary care. Mindfulness as a deautomation component (i.e., a skill to reduce automatic thoughts and behaviors) on the one hand, and as a moderator of behavior change on the other, could reduce impulsive eating in obese binge eaters $(\mathrm{H} 1)$ and increase their intrinsic motivation to exercise (H2), respectively. These changes in eating patterns and PA behaviors could regulate energy balance and lead to weight loss over one year (H3). Primary outcome measure will be change in impulsive eating from baseline to post-intervention. Secondary outcome measures will be change in motivational regulation to exercise, change in PA level (self-reported and objectively measured), change in mindfulness skills, change in plasma concentration of leptin, adiponectin, and brain-derived neurotrophic factor (BDNF), and change in food intake from baseline to post-intervention. Compliance with intervention will be an additional outcome measure that could moderate the observed effectiveness of the intervention.

\section{Design}

\section{METHODS AND DESIGN}

An open randomized controlled design will include obese patients randomly assigned to one of three conditions: a mindfulness-based intervention, a sham meditation intervention [29], or a treatment as usual (TAU). Randomization will be stratified on gender (male $v s$ female), BMI (BMI $<40 v s$ BMI $\geq 40$ ), and age (age $<46 v s$ age $\geq 46$ ). Forty-six years old has been used as a cut-off for age, as this was the mean age of patients at the Nutrition Pole in 2015. Participants in the three conditions benefit from TAU at the same Nutrition Department. Figure 1 displays the design of the intervention and a proposed participant flow through the trial. The 12-month study will examine the effects of the intervention on biomedical, behavioral, psychological, and adherence outcome measures at baseline, 1 month, 6 months, and post-intervention. Informed consent will be obtained from participants prior to inclusion in the study and anonymity during data analyses will be guaranteed. Ethical approval for the trial has been obtained from the Comité de Protection des Personnes Ile-deFrance 2 (approval number: ID-RCB 2015-A01370-49). The trial has been registered on ClinicalTrials.gov (NCT02571387). The study will take 24 months (12 months for patient recruiting, and 12 months for participation) and started in May 2016. 


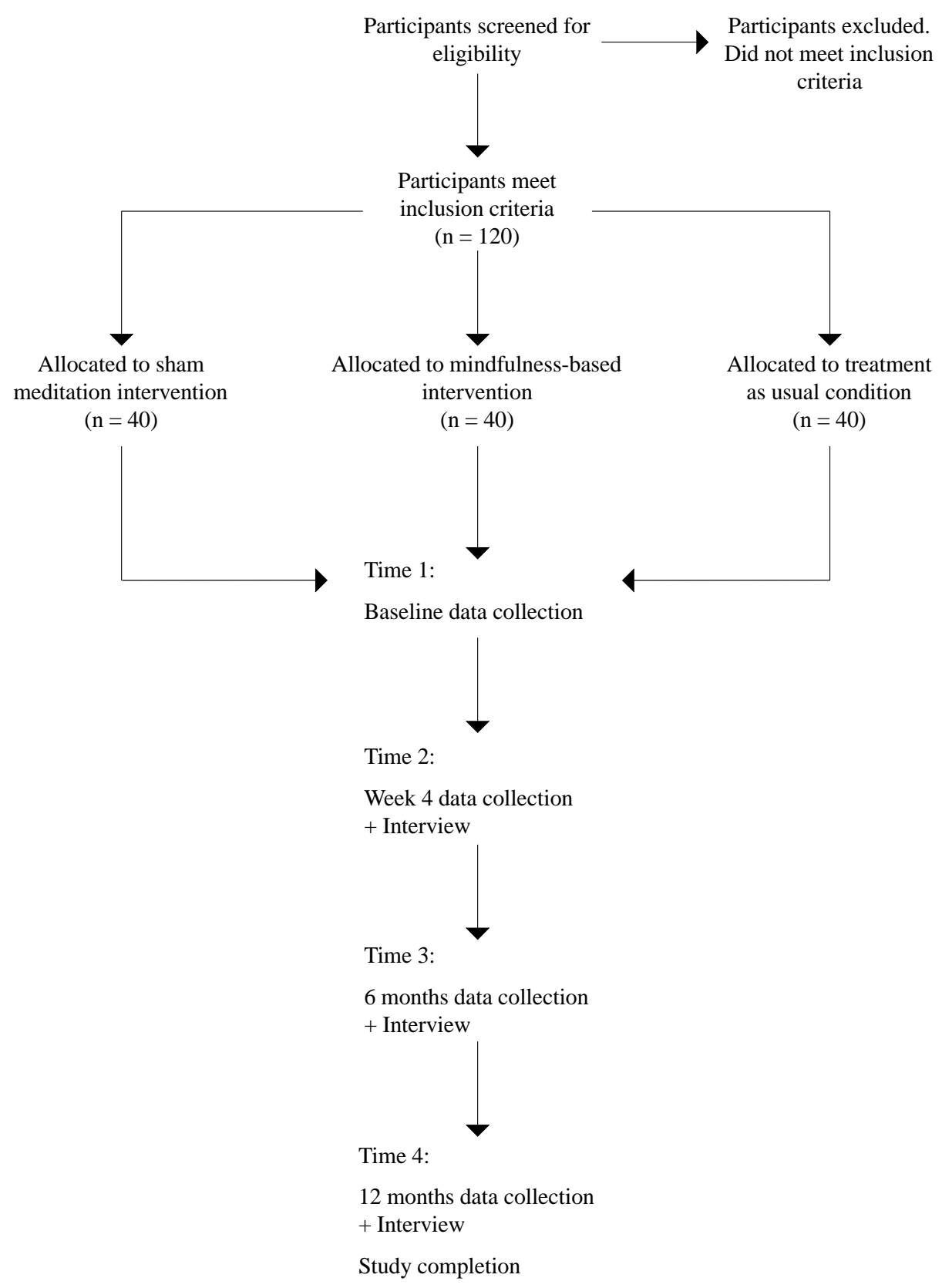

Figure 1. Predictive flow diagram of participation.

\section{Participants and procedure}

Participants will be recruited over 12 months at the Nutrition Departments of Ambroise Paré Hospital (Boulogne-Billancourt, France) and Georges-Pompidou European Hospital by a psychologist investigator in face-to-face semi-structured interviews. Patients will be systematically screened after their medical visit at the Nutrition Department. Inclusion criteria include obesity and BED. Diagnosis of BED follows the criteria of the Diagnostic and Statistical Manual of Mental Disorders (5 ${ }^{\text {th }}$ ed.; DSM-5) [30]. An investigator (accredited psychologist) will check the criteria during the inclusion interview, including: recurrent and persistent episodes of eating an abnormally large amount of food with a feeling of a lack of control during binge episodes associated with at least three of the five criteria (i.e., eating much more rapidly than normal, eating until feeling uncomfortably full, eating large amounts of food when not feeling physically hungry, eating alone because of being embarrassed by how much one is eating, or feeling disgusted with oneself, depressed, or very guilty after overeating), marked distress regarding binge eating, binge eating at least once a week during 
the last three months, and absence of regular compensatory behaviors (e.g., purging). Inclusion and exclusion criteria are displayed in Table 1.

Table 1. Inclusion and exclusion criteria.

\begin{tabular}{|c|c|}
\hline Inclusion criteria & Exclusion criteria \\
\hline $\begin{array}{l}\text { - } \text { Adult between } 18 \text { and } 65 \text { years of age } \\
\left.\text { - Obese (BMI } \geq 30 \mathrm{~kg} / \mathrm{m}^{2}\right) \\
\text { - Recruited from the Nutrition Departments } \\
\text { of Ambroise Paré Hospital and Georges- } \\
\text { Pompidou European Hospital } \\
\text { - Diagnosed with Binge-Eating Disorder } \\
\text { (BED) according to DSM5 criteria } \\
\text { - Informed consent provided during } \\
\text { inclusion interview }\end{array}$ & $\begin{array}{l}\text { - Already participating in a clinical trial } \\
\text { - Bariatric surgery patients (unless gastric } \\
\text { band removed at least } 3 \text { years before) } \\
\text { - Planned bariatric surgery } \\
\text { - Hypothalamic obesity (tumor or genetic) } \\
\text { - Pregnancy } \\
\text { - No Internet access at home } \\
\text { - Difficulties understanding French } \\
\text { - Patients under social protection } \\
\text { - Cognitive dysfunctions in understanding } \\
\text { simple guidelines provided by the } \\
\text { paramedical staff (e.g., diet or exercise } \\
\text { - guidelines) } \\
\text { - } \text { to hear auditory disorders that make it impossible } \\
\text { - Blurred vision that make it impossible to } \\
\text { use the Internet }\end{array}$ \\
\hline
\end{tabular}

The study will adopt a partial double-blind procedure so that participants assigned to an active condition (i.e., mindfulness or sham meditation), as well as researchers, are unaware of the intervention conditions. A research assistant uninformed about the aim of the study will ensure that each participant is provided with an identification number and randomly assigned to one of the three groups using a computer-generated list. An investigator will provide information and procedures to each participant according to the allocated condition.

Participants will need to provide written consent prior to participation. At baseline, 1month, 6-month, and post-intervention (12 months), participants will be asked to come back to the hospital for an appointment with an investigator. The purpose of these interviews is to keep participants motivated to participate, and to answer any questions they may have regarding technical and theoretical concerns. Prior to each appointment, participants will complete online questionnaires measuring behavioral and psychological outcomes, including declared PA levels, eating psychological processes, motivation toward exercise, anxiety and depression, and mindfulness skills. At baseline, 6-month intervention and post-intervention, participants will be required to report to the Nutrition Department to take blood samples and weight measurements.

Eligible patients recruited will be provided with (a) study information and (b) an online link to register anonymously to the study's online program and to fill in baseline assessments prior to their first online session. The study will be presented as complementary psychological training to participants assigned to the two active conditions (i.e., mindfulness and sham meditation), and as an understanding of psychological functioning of obese patients with BED to participants assigned to the inactive control group (i.e., TAU). Participants in the inactive control group will be informed at the end of the study that they can eventually benefit from one of the two active conditions. Likewise, at the end of the study, participants in the sham meditation condition will be informed that they can benefit from the mindfulness-based 
intervention. No measure will be made for participants allocated to a control condition who want to benefit from the tested intervention at the end of the study.

At the end of the baseline interview, participants will be provided with their next appointment time, several documents to help them register and use the online program, and a pedometer (Geonaute ONWalk 100). As participants in each condition benefit from the usual treatment for obesity, no additional information regarding diet, PA, or any education related to obesity will be provided in the intervention protocol.

Sample size has been determined by a power analysis, suitable to evaluate differences among the three conditions with statistical power set at $>.80$ and alpha set at $p<.05$. A moderate effect size is assumed, since a previous study that used a long-term self-help design including mindfulness training in obese individuals with BED [31] found moderate effect size on impulsive eating. Given the estimated effect size, the total sample size required post-test is 96; considering a potential $20 \%$ drop out rate based on previous trials [32], we plan to recruit 120 patients at baseline (i.e., 40 patients in each condition).

\section{Intervention}

\section{The treatment program.}

Participants in each group will benefit from usual nutrition care. TAU in the Nutrition Department includes grouped psycho-educational components relevant to obese individuals (e.g., diet, exercise, psychological processes, medical care) as well as individ ual care with nutritionists, dieticians, PA educators, and, if necessary, psychologists. Information regarding energy balance, medical comorbidities, diet, PA, psychological distress and eating disorders are provided to the patients. TAU in the Nutrition Department follows national guidelines from the French Authority for Health [33].

Complementary mindfulness-based intervention for obese individuals with BED will be held on a website designed for the MindOb protocol. Participants in the mindfulness condition will be asked to visit the website on a daily basis on their computer for one year, and listen to a 10-minute audio recorded mindfulness session. Four different sessions are made available to the participants: (a) awareness of breathing, (b) awareness of postures and bodily sensations, (c) acceptance of thoughts and emotions, and (d) awareness of thoughts and bodily sensations while executing five squats. During the first four weeks of the intervention (i.e., learning phase), participants will have restrained choice: session (a) during the first week, session (b) during the second, session (c) during the third, and session (d) during the fourth. After the learning phase, participants will have 11-month full access and free choice in selecting mindfulness sessions to listen (i.e., autonomous phase). Additionally, a 3-minute recording aiming at remembering the focus of the intervention (i.e., staying focused on the present moment non-judgmentally to reduced psychological distress) will remain available during the whole intervention.

The active control condition (i.e., sham meditation) follows the same procedure as the experimental condition (i.e., mindfulness-based intervention): a preliminary learning phase during the first four weeks followed by an autonomous phase during 11 months. Sham meditation audio sessions display (a) forest, (b) night, (c) beach, and (d) river background respectively. The only guideline during these recordings is provided during the first seconds of the session and is an invitation to "meditate". A 3-minute recording aiming at explaining the general principle of meditation will remain available during the whole intervention. Participants in the inactive control condition will benefit from TAU. Figure 2 displays the procedure for each condition. 


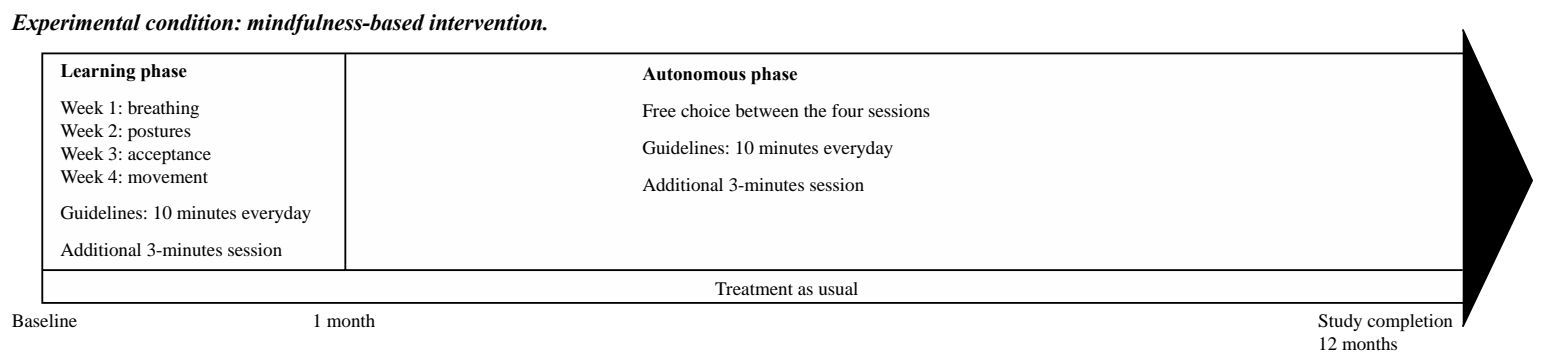

Active control condition: sham meditation.

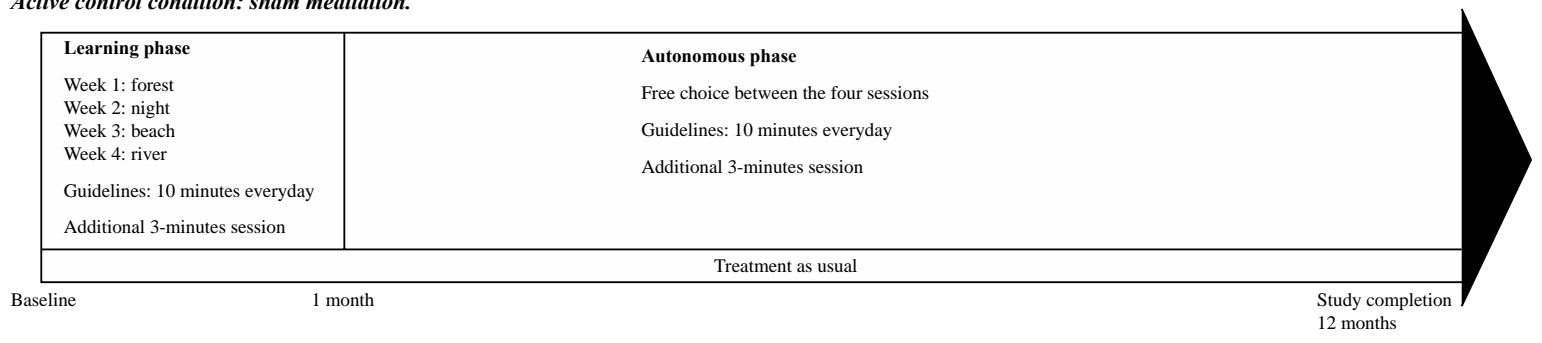

Inactive control condition: usual care in nutrition.

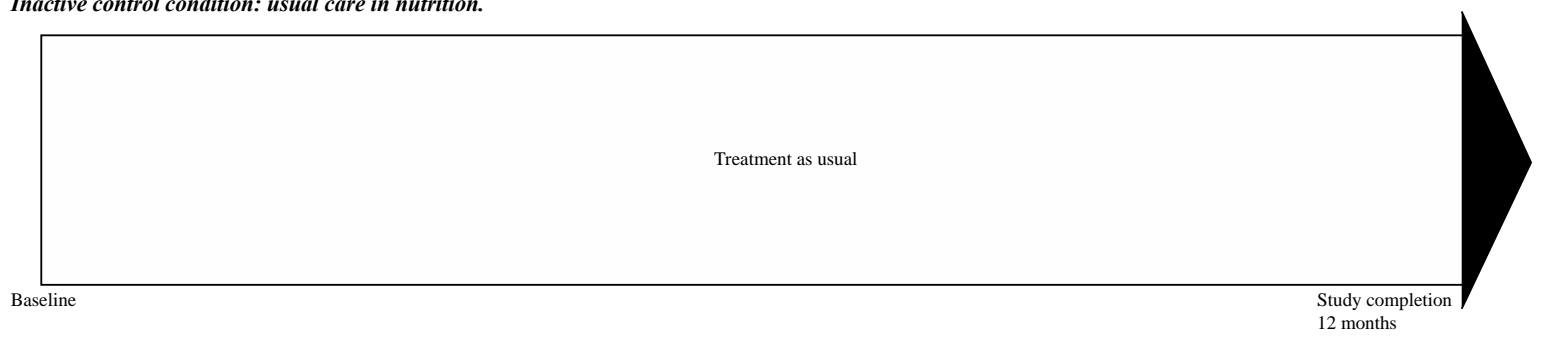

Figure 2. Procedure of intervention for each condition.

\section{Assessments}

Questionnaires measuring behavioral and psychological outcomes will be filled online, while socio-demographics, anthropometrics, clinical information and biomedical measurements will take place at the Nutrition Department, and compliance measurement will be extracted from the online program. Table 2 displays the measurement scheme. 
Table 2. Measurement scheme.

\begin{tabular}{|c|c|c|c|c|c|c|c|c|}
\hline Variable goal & Measure & Target & Screening & Baseline & During & 1 month & 6 months & 12 months \\
\hline Inclusion & DSM5 criteria & Binge eating disorder & $\mathrm{x}$ & & & & & \\
\hline Control variables & $\begin{array}{l}\text { Multiple-choice } \\
\text { questionnaire }\end{array}$ & $\begin{array}{l}\text { Sociodemographics, anthropometrics, } \\
\text { clinical information }\end{array}$ & & $\mathrm{x}$ & & & & \\
\hline \multirow[t]{4}{*}{ Biomedical outcomes } & BMI & Weight loss & & $\mathrm{x}$ & & & $\mathrm{x}$ & $\mathrm{x}$ \\
\hline & Leptin & Energy balance regulation & & $\mathrm{x}$ & & & $\mathrm{x}$ & $\mathrm{x}$ \\
\hline & Adiponectin & Metabolic inflammation & & $\mathrm{x}$ & & & $\mathrm{x}$ & $\mathrm{x}$ \\
\hline & BDNF & Hedonic eating and mood regulation & & $\mathrm{x}$ & & & $\mathrm{x}$ & $\mathrm{x}$ \\
\hline \multirow[t]{5}{*}{ Eating and PA outcomes } & TFEQ-R18 & Impulsive eating & & $\mathrm{x}$ & & $\mathrm{x}$ & $\mathrm{x}$ & $\mathrm{x}$ \\
\hline & Diet survey & Calories intake and diet balance & & $\mathrm{x}$ & & & $\mathrm{x}$ & $\mathrm{x}$ \\
\hline & BREQ-2 & Motivation toward exercise & & $\mathrm{x}$ & & $\mathrm{x}$ & $\mathrm{x}$ & $\mathrm{x}$ \\
\hline & IPAQ & Self-reported PA & & $\mathrm{x}$ & & $\mathrm{x}$ & $\mathrm{x}$ & $\mathrm{x}$ \\
\hline & Pedometers & PA level & & $\mathrm{x}$ & & & $\mathrm{x}$ & $\mathrm{x}$ \\
\hline Psychological measures & HADS & Psychological distress & & $\mathrm{x}$ & & $\mathrm{x}$ & $\mathrm{x}$ & $\mathrm{x}$ \\
\hline \multirow[t]{2}{*}{ Process measures } & MAAS & Dis positional mindfulness & & $\mathrm{x}$ & & $\mathrm{x}$ & $\mathrm{x}$ & $\mathrm{x}$ \\
\hline & DMRS & Daily mindful responding & & $\mathrm{x}$ & & & $\mathrm{x}$ & $\mathrm{x}$ \\
\hline Moderator & Server's data & Compliance & & & $\mathrm{x}$ & & & \\
\hline
\end{tabular}

DSM5: Diagnostic and statistical manual of mental disorders. BMI: Body mass index. BDNF: Brain-derived neurotrophic factor. PA: Physical activity. TFEQ-R18: Three-factor eating questionnaire. BREQ-2: Behavioral regulation in exercise questionnaire. IPAQ: International physical activity questionnaire. HADS: Hospital anxiety and depression scale. MAAS: Mindful attention awareness scale. DMRS: Daily mindful responding scale. 


\section{Sociodemographics, anthropometrics, and clinical information.}

Immediately after the inclusion interview, participants will be asked to fill out a form in which they provide socio-demographic information. The multiple-choice questionnaire includes themes such as gender, age, educational level, working and socio-economical situation, number of children, spirituality, medical comorbidities, age of onset of obesity, actual weight, time since first visit to the Nutrition Department, previous and current psychological care, prior knowledge of mindfulness, ease with new technologies, intentions to change, and motivation to participate in the study. These questions are adapted from previous and current sociological studies [34]. For each question, participants have the choice to state that they "do not want to answer" or "do not know the answer".

Biomedical outcomes.

A nurse blind to group allocation will measure height and weight, and will take blood samples from participants. Height will be measured at baseline (in meters), and weight (in kilograms) at baseline, six months, and 12 months. BMI is calculated from these measures at each measurement time $\left(\mathrm{BMI}=\right.$ weight $(\mathrm{kg}) /$ height $\left.(\mathrm{m})^{2}\right)$. Plasma concentration of adipokines (leptin and adiponectin) and BDNF will be collected at baseline, six months, and 12 months in $7 \mathrm{~mL}$ dry tubes. Two tubes will be collected (one for adipokines and one for $\mathrm{BDNF}$ ), centrifuged within 3 hours, stored at $-80{ }^{\circ} \mathrm{C}$, and sent every month to the laboratory for analysis.

Leptin and adiponectin are biomarkers of the level and quality of body fat. These hormones are secreted by adipose tissue. Plasma concentration of leptin is positively correlated with BMI, and plasma concentration of adiponectin is positively correlated with insulin-sensitivity $[35,36]$. BDNF is a factor that is highly concentrated in the hippocampus as well as in the cerebral cortex, and increases with growth and differentiation of new neurons and synapses. It has been shown that PA could increase BDNF secretion at the ARNm and protein levels in mices [37]. Moreover, BDNF shows negative correlations with depressed and manic moods, food intake, and obesity [38].

Eating and physical activity measurements.

The Three-Factor Eating Questionnaire (TFEQ-R18) [39] is an 18-item self-reported questionnaire that measures cognitive and behavioral components of eating: uncontrolled eating (items $1,4,5,7,8,9,13,14,17$; episodes of loss of control over eating), cognitive restraint (items $2,11,12,15,16,18$; control over food intake in order to influence body weight and shape), and emotional eating (items 3,6,10; overeating during dysphoric mood episodes). Items $1-17$ are rated on a 4-point Likert scale ranging from 1 to 4 ; and item 18 is rated on an 8-point Likert scale and coded as follow: $1-2$ as $1 ; 3-4$ as 2; 5-6 as 3; and 7-8 as 4. The latest validation study among obese individuals in Sweden showed internalconsistency reliability coefficients (Cronbach's alpha) for each of the three scales ranging from 0.78 to $0.94[39,40]$. Previous studies have shown that higher scores in the three factors are associated with higher BMI [41], and that cognitively restrained eating was negatively associated with uncontrolled eating in obese individuals [42]. Additionally, while women scored higher than men in cognitive restraint and emotional eating, no difference was found across gender according to uncontrolled eating scores [43]. Moreover, mindfulness-based interventions, as compared to a psycho-education group and an inactive control group, have been shown to reduce TFEQ-uncontrolled scores in obese individuals with BED [44].

Additionally, at baseline, 6 months, and 12 months, participants will be invited to complete online dietary recalls on a daily basis for 7 days. Each day, participants will have to state what they ate during each meal (i.e., breakfast, lunch, dinner) from a list of food items, providing the weight of each item. Data from two random weekdays and one random weekend day will be kept as a measure of food intake [45]. A dietician blind to allocation will check for invalid inputs. 
The Behavioral Regulation in Exercise Questionnaire (BREQ-2) [46] is a 19-item selfreported questionnaire measuring motivation regulation toward PA based on five subscales: amotivation (items 5, 9, 12, 19; lack of intention to engage in PA behavior), external regulation (items 1, 6, 11, 16; engaging in PA to satisfy external pressures or to earn external rewards), introjected regulation (items $2,7,13$; internalization of external controls applied through self-imposed pressures to avoid negative emotions or to maintain positive emotions), identified regulation (items $3,8,14,17$; conscious acceptance of PA as important for achieving self-valued outcomes), and intrinsic regulation (items 4, 10,15, 18; engaging in PA for its inherent satisfaction). The previous validation study showed internal-consistency reliability coefficients (Cronbach's alpha) for each of the five subscales ranging from 0.73 to 0.86 [46]. BREQ-2 is based on Self-Determination Theory (SDT) [47], which postulates that human behavior is motivated in order to satisfy basic psychological needs (autonomy, competence, and relatedness). Based on SDT, it is known that extrinsic motives (e.g., rewarded behavior) can lead to engagement in PA behavior, but intrinsic motives (e.g., for pleasure) are needed to maintain such behavior [48]. While mindfulness increases PA in obese and/or sedentary individuals, mechanisms of behavior change (e.g., motivation) induced by mindfulness-based interventions still lack evidence.

Self-reported PA levels will be assessed using the International Physical Activity Questionnaire (IPAQ-short form) [49]. The IPAQ provides information regarding participants' time spent (a) doing vigorous physical activities, (b) moderate physical activities, (c) walking, and (d) sitting during the past seven days (if not representative of the perceived average PA, participants are asked to think about the most representative past 7-day time frame). Scores are calculated using a coefficient of 8 to vigorous PA, 4 to moderate PA and 3.3 to walking; and translated into an overall energy expenditure expressed in Metabolic Equivalent Total (MET). In short, the IPAQ calculations are: Walking MET-min/week = 3.3 $\times$ walking minutes $\times$ walking days; Moderate MET-min/week $=4.0 \times$ moderate-intensity activity minutes $\times$ moderate days; Vigorous MET-min/week $=8.0 \times$ vigorous-intensity activity minutes $\times$ vigorous-intensity days. Total PA MET-min/week $=$ sum of Walking + Moderate + Vigorous MET minutes. The total MET-min per week will be used as a continuous indicator for PA [49].

PA will also be assessed with an objective measurement. Participants will be asked to wear pedometers during one full week at baseline, 6 months, and 12 months, and to report the distance $(\mathrm{km})$ and number of steps for each day of the week. Weekly distance and number of steps will be communicated to an investigator. Geonaute ONWalk100 is a three dimensional sensor aiming at measuring walking distance in everyday life. Participants can put the sensor in their pockets, bags, or clip it onto their belts. Participants will be provided with an additional kit of batteries in order to limit failures in usage during the 12-month participation. If participants want to use the sensor outside the scope of the study (i.e., for personal use), they will have to state the number of additional days they used it during their participation. In fact, the use of pedometers can lead to increased PA level and weight loss [50], and interfere with the effects of the tested intervention.

Psychological measures.

The Hospital Anxiety and Depression Scale (HADS) [51] is a 14-item assessment of anxiety (seven items) and depression (seven items) validated for individuals with somatic illnesses. Two factors (anxiety and depression) are rated on a 4-point Likert scale ranging from 0 to 3 and scored as the sum of items of each factor. In the literature, HADS has shown a mean internal-consistency reliability coefficient (Cronbach's alpha) for each of the two factors at 0.83 for anxiety and 0.82 for depression [52]. A previous review suggested that mindfulness decreases mood-related outcomes, such as anxiety and depression, with medium to large effect sizes (Cohen's $d$ ) at post-treatment [53]. 
Mindfulness skills will be assessed using two different scales to analyze whether the effects of the intervention could be explained by variations in mindfulness skills. The Mindful Attention Awareness Scale (MAAS) [54] measures self-reported dispositional mindfulness (i.e., the inherent ability of being mindful in everyday life) in 15 items rated on a 6-point Likert scale ranging from 1 ("almost always") to 6 ("almost never"). The score is coded as the mean of all items, ranging from 1 (low frequency of mindful states) to 6 (high frequency of mindful states). The validation of MAAS showed internal-consistency reliability coefficients (Cronbach's alpha) from 0.80 to 0.87 in seven different samples of participants [54]. The Daily Mindful Responding Scale (DMRS) [55] is a 4-item self-reported measure of mindful responding in the daily lives of individuals involved in mindfulness training. Participants will be asked to rate on a 10-point Likert scale ranging from 1 ("rarely") to 10 ("often") the extent to which they engaged in mindful responses when potential obstacles to being mindful arose during the day, on a daily basis and over one week. The previous validation study among participants undergoing mindfulness training showed internal-consistency reliability coefficients (Cronbach's alpha) of 0.83 for individual assessment [55].

Compliance-related outcomes.

Patients' compliance with the intervention will be measured for participants allocated to the mindfulness training group and the sham meditation group by dividing the number of sessions actually listened to by the number of days of participation. The number of sessions actually listened to is recorded at the participant level on the server data for each participant. Additionally, participants' speech from the 1 month, 6 month, and 12 month appointments will be analyzed in order to bring qualitative data to the objective compliance measurement (i.e., number of sessions listened) according to participants' beliefs, barriers and facilitators with regard to following the intervention. During the appointments, the investigator (an accredited psychologist) will type the main themes of patients' discourse into an online database made available for this study.

\section{Statistical analyses}

Data will be recorded online from the participants and via semi-structured interviews, and anonymous data will be extracted from the online database of the study at study completion. The primary analyses are aimed at showing the superiority of the mindfulness group compared to sham meditation and TAU groups in terms of post-intervention eating, PA and biomedical outcome measures. Sociodemographics, anthropometrics, clinical information, as well as psychological and compliance assessments will be used as control measurements for the effects of the intervention on eating, PA and biomedical outcomes. Secondary analyses will be conducted to test the dynamic processes of change for eating and PA outcomes during the intervention (i.e., at 1 month and 6 months).

Data for the primary analyses will be analyzed using repeated measure analysis of variance (ANOVA) with two factors (time $\times$ group) to compare psychological, biomedical, eating habit, PA, and weight-related outcomes at all time points among the three groups. Posthoc comparisons will be conducted using the Tukey-Kramer test with multiple hypothesis testing adjustment. In the absence of normality or equal variances, non-parametric equivalent analyses will be performed. Analysis of covariance (ANCOVA) modeling will be considered to account for differences between populations and increase power, taking into account baseline sociodemographics, anthropometrics, and clinical information.

Linear mixed models will estimate the predictive value of change in eating habit outcomes, PA outcomes, biomedical outcomes, and psychological outcomes on weight loss across groups. ANOVA between the models will be performed to compare these predictions between groups. Feasibility will be assessed by compared compliance related outcomes and drop-out/withdrawal rates within each intervention group. 
Appropriate data analysis techniques will be used to deal with potential missing data, which may include deletion, imputation, or inclusion of an indicator of missing values. All data will be tested for normality and equal variance using the Shapiro-Wilk and Bartlett tests. All values will be reported as mean \pm standard deviation, and nominally significant differences will be considered at $\mathrm{P}<.05$. All statistical analyses will be performed using $\mathrm{R}$ [56].

\section{DISCUSSION}

The aim of this 3-arm randomized controlled trial is to evaluate the effectiveness of a daily 12-month computerized mindfulness-based intervention in obese patients diagnosed with BED, as a complementary intervention to usual care in the Nutrition Department. The results of this study should be of interest to establish whether mindfulness could have a role to play in energy balance. It is hypothesized that improvement in mindfulness skills should decrease impulsive eating and increase intrinsic motivation to exercise, leading to larger longterm weight loss in participants who benefit from the tested intervention compared to participants allocated to active (i.e., sham meditation) and inactive (i.e., TAU) control groups.

Impulsive eating in obese individuals seems to be associated with difficulties in delaying gratification [57] and higher levels in urgency (i.e., difficulties in controlling impulses), lack of perseverance (i.e., difficulties in finishing what has been started), sensitivity to reward (i.e., doing things to be praised), and sensitivity to punishment (i.e., feeling afraid that something bad could happen) [58]. Moreover, randomized controlled trials testing the effectiveness of adapted mindfulness-based interventions for overweight and obese individuals showed results in reducing binge episodes and eating-related outcomes (e.g., hunger, restraint, lack of inhibition, craving) compared to an inactive control group postintervention (4 months) [44], and found that integrating mindfulness components into regular diet and PA interventions led to reduced reward-driven eating post-intervention (6 months) and greater weight-loss in the 12 and 18 month follow-ups than an active control group [59]. These primary investigations are in line with the MindOb protocol, arguing that mindfulness training could help individuals who seek medical advice to reduce body fat and manage their regular weight-loss treatments through changes in eating patterns (H1). However, none of these studies have investigated the effectiveness of a long-term (i.e., more than 6 months) mindfulness-based intervention taught as self-help and complementary to usual care in Nutrition.

Based on behavior change theories in the context of PA, studies investigating the role of mindfulness between (a) predictors of behavior change such as intentions [26] or motivational regulations [27] and (b) PA level, came to the conclusion that mindfulness skills exert a moderating effect ("moderating" in the sense of reducing extreme highs and lows, so that mindfulness prevents people from being extreme in either direction). Furthermore, mindfulness seems to facilitate the favorable processing of PA experiences by increasing individuals' satisfaction with PA [28]. However, these studies are preliminary findings using cross-sectional designs in healthy individuals. In the MindOb protocol, by intervening on the mindfulness variable in a mainly sedentary population, we would be able to validate previous models. Indeed, changes in mindfulness skills are expected to moderate the relationship between changes in intrinsic motivation to exercise and changes in PA levels (H2). Long-term participation in the mindfulness-based intervention, which should not only impact the motivation to exercise but also eating patterns, should lead to greater weight loss in the experimental group than in the control groups (H3).

While there is a growing interest in self-help designs for mindfulness-based interventions, previous studies have shown that the observed effects of these interventions are comparable to those of more classical designs (e.g., with face-to-face interviews) on 
depression, anxiety and mindfulness skills [60]. In patients diagnosed with BED, Grilo and Masheb [61] demonstrated that a 12-week self-help mindfulness-based intervention seems effective for BED symptoms but not for weight loss, with completion rates of $87 \%$ of the intervention for the self-help condition. A unique contribution of the present study would be the long-term ecological design of the mindfulness-based intervention. In fact, the 10 minute daily online self-help sessions appear to be easily integrated into patients' schedules. To test this assertion, investigators will collect qualitative data regarding the implementation of the sessions in patients' daily lives (e.g., where did they listen to the MP3s, at what time of the day) during the visits. Furthermore, assessing long-term psychological, behavioral and biomedical variables would highlight the processes linked to weight loss following a mindfulness-based intervention in obese patients with BED.

The number and repetition of assessments completed by participants could lead to measurement error and affirmation bias. However, it must be stressed that following the first appointment with the investigator (i.e., one month after their inclusion in the study) measurements and assessments will only be completed relatively infrequently (i.e., twice at six-month intervals). Furthermore, given the duration of the participation, we can expect that only motivated and compliant patients will be likely to complete the study, which would introduce bias to the findings. However, an average of $73 \%$ of individuals participating in self-help mindfulness-based interventions complete post-intervention assignments, and complete $69 \%$ of these interventions [60]. These findings support the evidence that mindfulness can be taught with a self-help design, with completion rates - and effect sizes close to those of non-self-help interventions. Another limitation would be that participants would have no benefit from psychoeducation on mindfulness techniques or BED. This choice has been made in order to observe the effects of the tested intervention solely (i.e., in terms of compliance to the treatment), and to limit the time spent with a therapist, keeping administration and personnel costs low.

Further investigations regarding the cognitive processes following mindfulness-based interventions would be needed in order to explain why patients would be less impulsive in their eating behaviors and more intrinsically motivated to increase their PA behaviors. Moreover, the same intervention could be tested with deeper individual guidance and psychoeducational group meetings aiming at enhancing treatment compliance and effectiveness. 


\section{References}

[1] M. Ng, T. Fleming, M. Robinson, B. Thomson, N. Graetz, C. Margono, E.C. Mullany, S. Biryukov, C. Abbafati, S.F. Abera, J.P. Abraham, N.M.E. Abu-Rmeileh, T. Achoki, F.S. AlBuhairan, Z.A. Alemu, R. Alfonso, M.K. Ali, R. Ali, N.A. Guzman, W. Ammar, P. Anwari, A. Banerjee, S. Barquera, S. Basu, D.A. Bennett, Z. Bhutta, J. Blore, N. Cabral, I.C. Nonato, J.-C. Chang, R. Chowdhury, K.J. Courville, M.H. Criqui, D.K. Cundiff, K.C. Dabhadkar, L. Dandona, A. Davis, A. Dayama, S.D. Dharmaratne, E.L. Ding, A.M. Durrani, A. Esteghamati, F. Farzadfar, D.F.J. Fay, V.L. Feigin, A. Flaxman, M.H. Forouzanfar, A. Goto, M.A. Green, R. Gupta, N. Hafezi-Nejad, G.J. Hankey, H.C. Harewood, R. Havmoeller, S. Hay, L. Hernandez, A. Husseini, B.T. Idrisov, N. Ikeda, F. Islami, E. Jahangir, S.K. Jassal, S.H. Jee, M. Jeffreys, J.B. Jonas, E.K. Kabagambe, S.E.A.H. Khalifa, A.P. Kengne, Y.S. Khader, Y.-H. Khang, D. Kim, R.W. Kimokoti, J.M. Kinge, Y. Kokubo, S. Kosen, G. Kwan, T. Lai, M. Leinsalu, Y. Li, X. Liang, S. Liu, G. Logroscino, P.A. Lotufo, Y. Lu, J. Ma, N.K. Mainoo, G.A. Mensah, T.R. Merriman, A.H. Mokdad, J. Moschandreas, M. Naghavi, A. Naheed, D. Nand, K.M.V. Narayan, E.L. Nelson, M.L. Neuhouser, M.I. Nisar, T. Ohkubo, S.O. Oti, A. Pedroza, D. Prabhakaran, N. Roy, U. Sampson, H. Seo, S.G. Sepanlou, K. Shibuya, R. Shiri, I. Shiue, G.M. Singh, J.A. Singh, V. Skirbekk, N.J.C. Stapelberg, L. Sturua, B.L. Sykes, M. Tobias, B.X. Tran, L. Trasande, H. Toyoshima, S. van de Vijver, T.J. Vasankari, J.L. Veerman, G. Velasquez-Melendez, V.V. Vlassov, S.E. Vollset, T. Vos, C. Wang, X. Wang, E. Weiderpass, A. Werdecker, J.L. Wright, Y.C. Yang, H. Yatsuya, J. Yoon, S.-J. Yoon, Y. Zhao, M. Zhou, S. Zhu, A.D. Lopez, C.J.L. Murray, E. Gakidou, Global, regional, and national prevalence of overweight and obesity in children and adults during 1980-2013: A systematic analysis for the Global Burden of Disease Study 2013, The Lancet 384(9945) (2014) 766-781.

[2] World Health Organization, Global recommendations on physical activity for health, 2010.

[3] J.I. Hudson, E. Hiripi, H.G.J. Pope, R.C. Kessler, The prevalence and correlates of eating disorders in the national comorbidity survey replication, Biol Psychiatry 61 (2007) 348-358. [4] A.J. Hill, Obesity and eating disorders, Obes Rev 8(Suppl 1) (2007) 151-155.

[5] S.J. Street, J.C.K. Wells, A.P. Hills, Windows of opportunity for physical activity in the prevention of obesity, Obes Rev 16(10) (2015) 857-870.

[6] M. Fogelholm, K. Kukkonen-Harjula, Does physical activity prevent weight gain - A systematic review, Obes Rev 1(2) (2000) 95-111.

[7] M.A. Cornier, E.L. Melanson, A.K. Salzberg, J.L. Bechtell, J.R. Tregellas, The effects of exercise on the neuronal response to food cues, Physiol Behav 105(4) (2012) 1028-1034.

[8] J. Kabat-Zinn, Full catastrophe living: Using the wisdom of your body and mind to face stress, pain, and illness, Delta Trade Paperbacks, New York, NY, 1990.

[9] K.C. Roberts, S. Danoff-Burg, Mindfulness and health behaviors: Is paying attention good for you?, J Am Coll Health 59(3) (2010) 165-173.

[10] K.M. Godfrey, L.C. Gallo, N. Afari, Mindfulness-based interventions for binge eating: A systematic review and meta-analysis, J Behav Med 38(2) (2014) 348-362.

[11] J. Godsey, The role of mindfulness based interventions in the treatment of obesity and eating disorders: An integrative review, Complement Ther Med 21(4) (2013) 430-439.

[12] S.N. Katterman, B.M. Kleinman, M.M. Hood, L.M. Nackers, J.A. Corsica, Mindfulness meditation as an intervention for binge eating, emotional eating, and weight loss: A systematic review, Eat Behav 15(2) (2014) 197-204.

[13] G.A. O'Reilly, L. Cook, D. Spruijt-Metz, D.S. Black, Mindfulness-based interventions for obesity-related eating behaviours: A literature review, Obes Rev 15(6) (2014) 453-461. [14] K.L. Olson, C.F. Emery, Mindfulness and weight loss: A systematic review, Psychosom Med 77(1) (2015) 59-67. 
[15] M.M.P. Demarzo, A. Cebolla, J. Garcia-Campayo, The implementation of mind fulness in healthcare systems: A theoretical analysis, Gen Hosp Psychiatry 37(2) (2015) 166-171. [16] V.S. Malik, W.C. Willett, F.B. Hu, Global obesity: Trends, risk factors and policy implications, Nat Rev Endocrinol 9 (2013) 13-27.

[17] A. Ruffault, S. Grégoire, K. Hendrickson, C. Flahault, Cognitive behavioral therapy with daily mindfulness training: a case report of comorbid binge eating and bipolar disorders, Mindfulness 7(2) (2016) 573-576.

[18] M.R. Yeomans, M. Leitch, S. Mobini, Impulsivity is associated with the disinhibition but not restraint factor from the Three Factor Eating Questionnaire, Appetite 50 (2008) 469-476.

[19] G. Gerlach, S. Herpertz, S. Loeber, Personality traits and obesity: A systematic review, Obes Rev 16(1) (2015) 32-63.

[20] K. Schag, J. Schönleber, M. Teufel, S. Zipfel, K. Giel, Food-related impulsivity in obesity and Binge Eating Disorder - A systematic review, Obes Rev 14(6) (2013) 477-495. [21] J. Lacaille, J. Ly, N. Zacchia, S. Bourkas, E. Glaser, B. Knäuper, The effects of three mindfulness skills on chocolate cravings, Appetite 76 (2014) 101-112.

[22] K.L. Hendrickson, E.B. Rasmussen, Effects of mindful eating training on delay and probability discounting for food and money in obese and healthy-weight individuals, Behav Res Ther 51(7) (2013) 399-409.

[23] P. Lattimore, N. Fisher, P. Malinowski, A cross-sectional investigation of trait disinhibition and its association with mindfulness and impulsivity, Appetite 56 (2011) 241 248.

[24] C.S. Ulmer, B.A. Stetson, P.G. Salmon, Mindfulness and acceptance are associated with exercise maintenance in YMCA exercisers, Behav Res Ther 48(8) (2010) 805-809.

[25] M.L. Butryn, E. Forman, K. Hoffman, J. Shaw, A. Juarascio, A pilot study of acceptance and commitment therapy for promotion of physical activity, J Phys Act Health 8(4) (2011) 516-522.

[26] N.L.D. Chatzisarantis, M.S. Hagger, Mindfulness and the intention-behavior relationship within the theory of planned behavior, Pers Soc Psychol Bull 33(5) (2007) 663-676.

[27] A. Ruffault, M. Bernier, N. Juge, J.F. Fournier, Mindfulness may moderate the relationship between intrinsic motivation and physical activity: A cross-sectional study, Mindfulness 7(2) (2016) 445-452.

[28] K.E. Tsafou, D.T.D. DeRidder, R. vanEe, J.P.W. Lacroix, Mindfulness and satisfaction in physical activity: A cross-sectional study in the Dutch population, J Health Psychol (2015) $1-11$.

[29] F. Zeidan, S.K. Johnson, N.S. Gordon, P. Goolkasian, Effects of brief and sham mindfulness meditation on mood and cardiovascular variables, J Altern Complem Med 16(8) (2010) 867-873.

[30] American Psychiatric Association, Diagnostic and statistical manual of mental disorders, 5th ed., American Psychiatric Association, Washington, DC, 2013.

[31] I. Carrard, C. Crépin, P. Rouget, T. Lam, M. Van der Linden, A. Golay, Acceptance and efficacy of a guided internet self-help treatment program for obese patients with binge eating disorder, Clin Pract Epidemiol Ment Health 7 (2011) 8-18.

[32] M. Buhrman, A. Skoglund, J. Husell, K. Bergström, T. Gordh, T. Hursti, N. Bendelin, T. Furmark, G. Andersson, Guided internet-delivered acceptance and commitment therapy for chronic pain patients: A randomized controlled trial, Behav Res Ther 51(6) (2013) 307-315. [33] Haute Autorité de Santé, Surpoids et obésité de l'adulte : prise en charge médicale de premier recours <http://www.has-sante.fr>, 2011 (accessed 2015/10/27.).

[34] European Values Study, European Values Study 2008: Integrated Dataset (EVS 2008), GESIS Data Archive, Cologne, Germany, 2011. 
[35] A.R. de Almeida, S. Monte-Alegre, M.B. Zanini, A.L. Souza, M. Etchebehere, J.A.R. Gontijo, Association between prehypertension, metabolic and inflammatory markers, decreased adiponectin and enhanced insulinemia in obese subjects, Nutr Metab 11(25) (2014) $1-11$.

[36] K. Nakamura, J.J. Fuster, K. Walsh, Adipokines: A link between obesity and cardiovascular disease, J Cardiol 63(4) (2014) 250-259.

[37] S.A. Neeper, F. Gomezpinilla, J. Choi, C. Cotman, Exercice and brain neurotrophins, Nature 373 (1995) 109.

[38] J.W. Cordeira, L. Frank, M. Sena-Esteves, E.N. Pothos, M. Rios, Brain-Derived Neurotrophic Factor regulates hedonic feeding by acting on the mesolimbic dopamine system, J Neurosci 30(7) (2010) 2533-2541.

[39] J. Karlsson, L.O. Persson, L. Sjostrom, M. Sullivan, Psychometric properties and factor structure of the Three-Factor Eating Questionnaire (TFEQ) in obese men and women: Results from the Swedish Obese Subjects (SOS) study, Int J Obes 24 (2000) 1715-1725.

[40] J.C. Cappelleri, A.G. Bushmakin, R.A. Gerber, N.K. Leidy, C.C. Sexton, M.R. Lowe, J. Karlsson, Psychometric analysis of the Three-Factor Eating Questionnaire-R21: Results from a large diverse sample of obese and non-obese participants, Int J Obes 33(6) (2009) 611-620. [41] K. Keskitalo, H. Tuorila, T.D. Spector, L.F. Cherkas, A. Knaapila, J. Kaprio, K. Silventoinen, M. Perola, The Three-Factor Eating Questionnaire, body mass index, and responses to sweet and salty fatty foods: A twin study of genetic and environmental associations, Am J Clin Nutr 88(2) (2008) 263-271.

[42] H. Konttinen, A. Haukkala, S. Sarlio-Lähteenkorva, K. Silventoinen, P. Jousilahti, Eating styles, self-control and obesity indicators: The moderating role of obesity status and dieting history on restrained eating, Appetite 53(1) (2009) 131-134.

[43] B. de Lauzon, M. Romon, V. Deschamps, L. Lafay, J.-M. Borys, J. Karlsson, P. Ducimetière, M.A. Charles, the Fleurbaix Laventie Ville Sante (FLVS) Study Group, The Three-Factor Eating Questionnaire-R18 is able to distinguish among different eating patterns in a general population, J Nutr 134(9) (2004) 2372-2380.

[44] J. Kristeller, R.Q. Wolever, V. Sheets, Mindfulness-Based Eating Awareness Training (MB-EAT) for binge eating: A randomized clinical trial, Mindfulness 5(3) (2014) 282-297. [45] I.H.E. Rutishauser, Dietary intake measurements, Public Health Nutr 8(7A) (2005) 11001107.

[46] D. Markland, V. Tobin, A modification to the Behavioural Regulation in Exercise Questionnaire to include an assessment of amotivation, J Sport Exerc Psychol 26(2) (2004) 191-196.

[47] E.L. Deci, R.M. Ryan, Intrinsic motivation and self-determination in human behavior, Plenum, New York, NY, 1985.

[48] M. Hagger, N. Chatzisarantis, Intrinsic motivation and self-determination in exercise and sport, Human Kinetics, Champaign, IL, 2007.

[49] C.L. Craig, A.L. Marshall, M. Sj??Str??M, A.E. Bauman, M.L. Booth, B.E. Ainsworth, M. Pratt, U. Ekelund, A. Yngve, J.F. Sallis, P. Oja, International Physical Activity Questionnaire: 12-country reliability and validity, Med Sci Sport Exer 35(8) (2003) 13811395.

[50] D.M. Bravata, C. Smith-Spangler, V. Sundaram, A.L. Gienger, N. Lin, R. Lewis, C.D. Stave, I. Olkin, J.R. Sirard, Using pedometers to increase physical activity and improve health: A systematic review, JAMA 298(19) (2007) 2296-2304.

[51] A.S. Zigmond, R.P. Snaith, The Hospital Anxiety and Depression Scale, Acta Psychiatr Scand 67 (1983) 361-370.

[52] I. Bjelland, A.A. Dahl, T.T. Haug, D. Neckelmann, The validity of the Hospital Anxiety and Depression Scale: An updated literature review, J Psychosom Res 52(2) (2002) 69-77. 
[53] R.A. Baer, Mindfulness training as a clinical intervention: A conceptual and empirical review, Clin Psychol: Sci Pr 10(2) (2003) 125-143.

[54] K.W. Brown, R.M. Ryan, The benefits of being present: Mindfulness and its role in psychological well-being, J Pers Soc Psychol 84(4) (2003) 822-848.

[55] J. Lacaille, G. Sadikaj, M. Nishioka, J. Flanders, B. Knäuper, Measuring mindful responding in daily life: Validation of the Daily Mindful Responding Scale (DMRS), Mindfulness (2015).

[56] R Core Team, R: A language and environment for statistical computing, R Foundation for Statistical Computing, Vienna, Austria, 2013.

[57] L.H. Epstein, S.J. Salvy, K.A. Carr, K.K. Dearing, W.K. Bickel, Food reinforcement, delay discounting and obesity, Physiol Behav 100 (2010) 438-445.

[58] O. Mobbs, C. Crépin, C. Thiéry, A. Golay, M. Van der Linden, Obesity and the four facets of impulsivity, Patient Educ Couns 79 (2010) 372-377.

[59] A.E. Mason, E.S. Epel, K. Aschbacher, R.H. Lustig, M. Acree, J. Kristeller, M. Cohn, M. Dallman, P.J. Moran, P. Bacchetti, Barbara Laraia, F.M. Hecht, J. Daubenmier, Reduced reward-driven eating accounts for the impact of a mindfulness-based diet and exercise intervention on weight loss: Data from the SHINE randomized controlled trial, Appetite (2016).

[60] K. Cavanagh, C. Strauss, L. Forder, F. Jones, Can mindfulness and acceptance be learnt by self-help?: A systematic review and meta-analys is of mindfulness and acceptance-based self-help interventions, Clin Psychol Rev 34 (2014) 118-129.

[61] C.M. Grilo, R.M. Masheb, A randomized controlled comparison of guided self-help cognitive behavioraltherapy and behavioralweight loss for binge eating disorder, Behav Res Ther 43 (2005) 1509-1525. 